\title{
ARTICLE
}

\section{Neutron and Gamma-ray Dose Evaluation on Accelerator Neutron Source using p-Li Reaction for BNCT}

\author{
Hiroyuki MIYAMARU*and Isao MURATA \\ Division of Electrical, Electronic and Information Engineering, \\ Graduate School of Engineering Osaka University, Osaka 565-0871, Japan
}

\begin{abstract}
A compact moderator assembly for an accelerator neutron source using the $\mathrm{p}$-Li reaction was designed to examine the performance of epithermal neutron generation and the shielding of fast neutrons and secondary gamma rays. Neutron and gamma-ray transport in the assembly were simulated using the Monte Carlo code MCNP4C. The primary neutron intensity at a beam target was estimated from the nuclear reaction data using the DROSG-2000 code. The use of heavy water, Fluental ${ }^{\mathrm{TM}}$, carbon, and beryllium was investigated as moderators. The epithermal and fast neutron intensities at the moderator assembly exit were evaluated. The dimensions of the moderator were limited because the distance between the beam target and an irradiation position should be less to obtain sufficient epithermal neutrons for clinical treatment. Our results indicated that the use of heavy water as a moderator was suitable for epithermal neutron production with higher flux and decreasing number of fast neutrons. Gamma rays generated directly at the target were suppressed by using a thick bismuth block placed along the beam axis. Secondary gamma rays generated by the cadmium sheet used for shielding thermal neutrons were found to contribute significantly to the increase in the gamma-ray dose, which became almost equal to that by fast neutrons.
\end{abstract}

KEY WORDS: BNCT, accelerator neutron source, gamma ray, p-Li reaction, Monte Carlo simulation

\section{Introduction}

An accelerator neutron source using nuclear reaction has recently been proposed and developed for application to boron neutron capture therapy ${ }^{1), 2}(\mathrm{BNCT})$. The accelerator neutron source using the ${ }^{7} \mathrm{Li}(\mathrm{p}, \mathrm{n})^{7} \mathrm{Be}$ reaction is particularly promising owing to its high neutron production yield and higher feasibility. Nonetheless, the neutron intensity in the accelerator neutron source is observed to be lower than that in a nuclear reactor due to the limitation of the allowable beam current. In order to obtain sufficient epithermal $(0.5-10 \mathrm{keV})$ neutron beam intensity for clinical treatment, the distance between an irradiation position and a beam target should be reduced. Therefore, the size of a moderator assembly is spatially limited and radiation shielding may be insufficient. In addition to the fast neutrons, secondary gamma rays generated in the assembly also contribute to the radiation dose at the irradiation position. Appropriate shielding is thus required in this limited space to minimize unwanted radiation exposure during clinical treatment. The assembly design should thus provide sufficient shielding against fast neutrons and gamma rays while maintaining high epithermal neutron intensity.

In this study, the transport profiles of neutrons and gamma rays in a compact assembly designed were examined using the Monte Carlo code MCNP4C ${ }^{3)}$. The feasibility of a neutron source using the $\mathrm{p}$-Li reaction is discussed.

\section{Material and Methods}

\footnotetext{
*Corresponding Author, Tel. +81-6-6879-7894, Fax. +81-6-6879-7899, E-mail: miyamaru@eei.eng.osaka-u.ac.jp
}

A moderator assembly was designed to obtain high intensity of epithermal neutrons. Figure 1 shows a cross-sectional view of the assembly geometry. A stainless steel pipe is used as a beam port (diameter: $5 \mathrm{~cm}$ ) and set at the center. A 3-mm-thick lithium target is positioned within the end of the pipe. A conical moderator is placed at the end of the beam pipe. The pipe and the moderator are enclosed within a cylindrical carbon reflector (diameter: $60 \mathrm{~cm}$ ). A 1 -mm-thick cadmium sheet is positioned such that it shields the thermal neutron component. A 1-cm-thick bismuth (Bi) plate is placed at the end of the assembly to shield gamma rays. Additionally, a 3-cm-thick cylindrical bismuth block is placed at the center to further shield the gamma-ray dose at the irradiation position. The arrangement of the moderator and the gamma-ray shielding materials was optimized by comparing the calculation results of the neutron and gamma-ray intensities under various conditions. In this study, we investigated the use of heavy water $\left(\mathrm{D}_{2} \mathrm{O}\right)$, Fluental $^{\mathrm{TM}}$, carbon $(\mathrm{C})$, and beryllium $(\mathrm{Be})$ as moderators. Fluental $^{\mathrm{TM}}$ is a composite material of $\mathrm{Al}(30 \%), \mathrm{AlF}_{3}(69 \%)$, and $\operatorname{LiF}(1 \%)$ and is used as a moderator in some BNCT facilities $^{4}$.

The transport profiles of neutrons and gamma rays were calculated from the target position through the moderator assembly by using Monte Carlo code MCNP4C accompanied with JENDL $3.2^{5)}$ nuclear data library. Neutron and gamma-ray fluxes at the assembly exit were evaluated using the surface flux tally in the calculation. This evaluation position was set to be a position along the beam axis that was $25 \mathrm{~cm}$ away from the target as indicated in Fig.1. In order to evaluate neutron and gamma-ray radiation doses to a human body briefly, a cubic water phantom $\left(1000 \mathrm{~cm}^{3}\right)$ was 
placed at the evaluation position when calculating an absorbed dose for each fast neutrons and gamma rays. The dose calculation was performed with the energy deposition tally. To accomplish statistical error less than $5 \%$ for every output tallies, more than 10 million of histories were done in every calculation conditions examined.

The primary neutron yield by the ${ }^{7} \mathrm{Li}(\mathrm{p}, \mathrm{n})^{7} \mathrm{Be}$ reaction was estimated using DROSG-2000 ${ }^{6}$ code to be approximately $5 \times$ $10^{12} \mathrm{n} / \mathrm{s}$ assuming a beam energy and current of $2.3 \mathrm{MeV}$ and $10 \mathrm{~mA}$, respectively. This value is similar to the one calculated by other researchers ${ }^{7)}$. This neutron intensity is barely satisfactory in terms of the criteria for clinical use an epithermal neutron flux of $10^{9} \mathrm{n} / \mathrm{cm}^{2}$ at an irradiation position located $25-30 \mathrm{~cm}$ away from the target. The subsequent calculations of neutron flux and dose estimation are based on the assumption of this primary neutron intensity at the target. The angular dependencies of both the neutron energy and the yield were evaluated every $10^{\circ}$ and these values are considered subsequently.

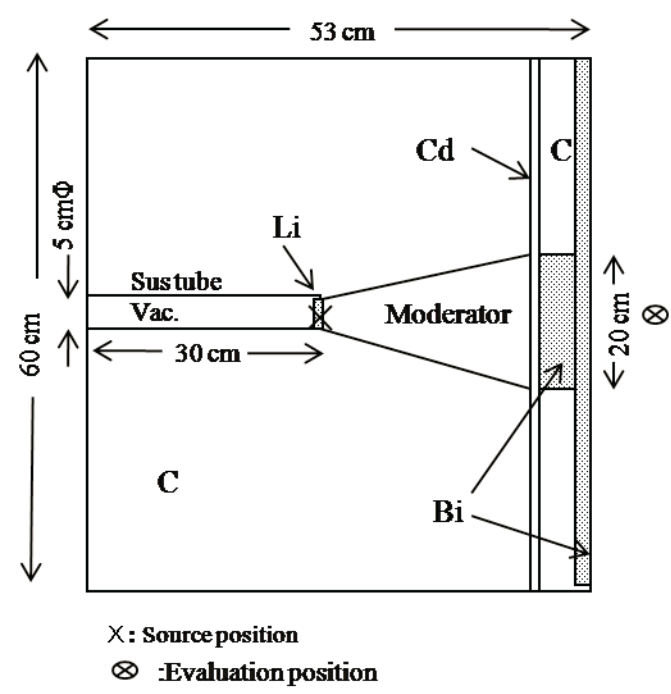

Fig. 1 Cross-sectional view of the moderator assembly for MCNP calculation.

\section{Results and discussion}

\section{Moderator Characteristics}

Figure 2 shows a comparison of the neutron energy spectra for each moderator material. When using Fluental ${ }^{\mathrm{TM}}$, the neutron flux over $1 \mathrm{keV}$ is greater than those of other materials. Since the main component of Fluental ${ }^{\mathrm{TM}}$ is aluminum and fluorine, energy deposition of the fast neutrons to the moderator in one collision becomes smaller rather than other materials examined. This result indicates that the quantity of Fluental ${ }^{\mathrm{TM}}$ is insufficient in such a limited moderator space to moderate fast neutrons. In the epithermal range, a higher flux can be obtained using heavy water, while the flux is very low when beryllium is used. The use of carbon provides a higher neutron flux in the fast energy range but it is lower in the epithermal range. In all the conditions, thermal neutron intensities were approximately two orders of magnitude lower than those of the epithermal flux, and they were successfully suppressed by using a cadmium sheet.

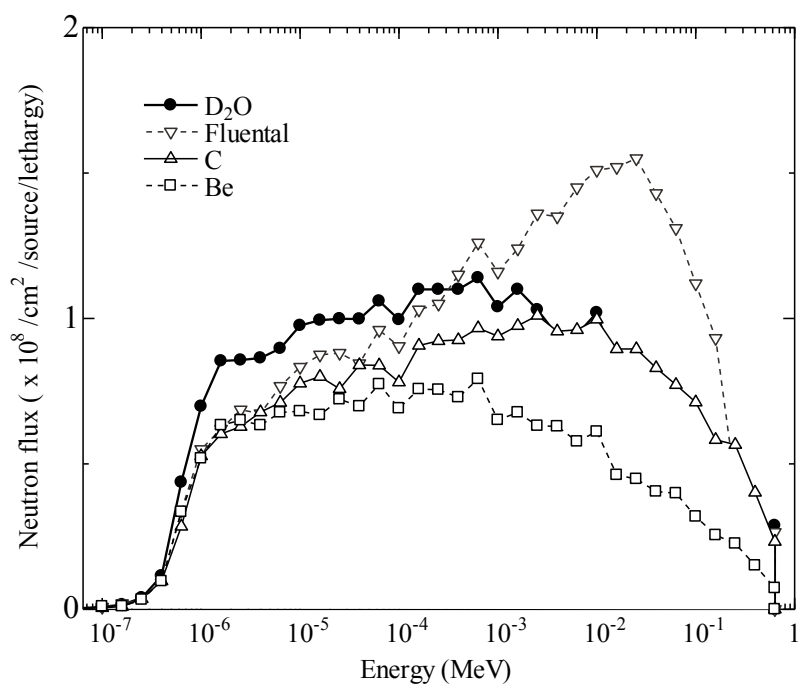

Fig. 2 Comparison of the neutron energy spectra for each moderator material.

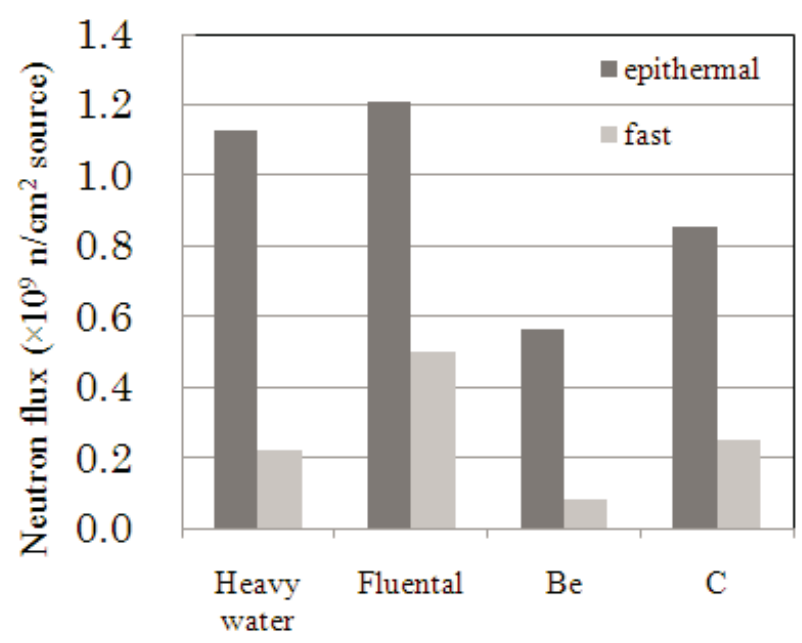

Fig.3 Fast and epithermal neutron fluxes for each moderator material.

Figure 3 shows a comparison of fast and epithermal neutron fluxes at the evaluation position for each moderator material. The epithermal neutron flux corresponds to the integral of the energy range from $0.5 \mathrm{eV}$ to $10 \mathrm{keV}$ in Fig. 2 and the fast neutron flux is the one over $10 \mathrm{keV}$. It is observed that the epithermal neutron fluxes in both cases of carbon and beryllium moderators do not exceed the value of $1 \times 10^{9} \mathrm{n} / \mathrm{cm}^{2}$ corresponding with the intensity criterion for clinical use. The use of Fluental ${ }^{\mathrm{TM}}$ provides sufficient epithermal neutrons, but the fast neutron flux is approximately 2.5 times larger than that in the case of heavy water. 
The characteristics of the neutron transport profile of each moderator material are summarized in Table. 1. The value of the fast neutron dose $\left(D_{\text {fast }}\right)$ divided by the epithermal neutron flux $\left(\Phi_{\text {epi }}\right)$ was evaluated. From the view of the radiation safety, this value is often referred for BNCT and a smaller value indicates excellent performance of the accelerator neutron source. As shown in the table, the heavy water moderator is observed to have a lower value than Fluental $^{\mathrm{TM}}$. Although the beryllium moderator also has a lower value, it cannot be used because of the low epithermal neutron intensity. Therefore, we concluded that the use of heavy water is suitable for our designed moderator assembly.

Table. 1 Epithermal neutron flux and the value of fast neutron dose divided by epithermal neutron flux.

\begin{tabular}{|c|c|c|c|c|}
\hline & $\mathrm{D}_{2} \mathrm{O}$ & Fluental $^{\mathrm{TM}}$ & $\mathrm{Be}$ & $\mathrm{C}$ \\
\hline $\begin{array}{c}\Phi_{\text {epi }} \\
{\left[\times 10^{9} / \mathrm{cm}^{2} / \text { source }\right]}\end{array}$ & 1.1 & 1.2 & 0.6 & 0.9 \\
\hline $\begin{array}{c}\mathrm{D}_{\text {fast }} / \Phi_{\mathrm{epi}} \\
{\left[\times 10^{-12} \mathrm{~Gy} \mathrm{~cm}^{2}\right]}\end{array}$ & 1.1 & 1.5 & 0.6 & 1.8 \\
\hline
\end{tabular}

\section{Gamma-ray dose}

The influence of gamma rays generated in the moderator assembly was evaluated only in the case of the heavy water moderator. In our previous study ${ }^{8)}$, we found that an intense emission of 478-keV gamma rays simultaneously occurred during the production of fast neutrons by the $\mathrm{p}-\mathrm{Li}$ reaction. This gamma-ray emission originated from $\mathrm{Li}\left(\mathrm{p}, \mathrm{p}^{\prime}\right) \mathrm{Li}$ inelastic scattering and it is not dependent on the materials surrounding the target. In our calculation, the gamma-ray intensity of this direct component was estimated to be approximately $1 \times 10^{12} / 10 \mathrm{~mA}$ at the target position. To reduce the gamma-ray dose effectively, a thick bismuth block was placed along the beam axis. In this case, the dose contribution of this direct component became negligible, but other secondary gamma rays generated by neutrons in the surrounding materials contributed to an increase in the dose at the evaluation position. Table 2 shows the epithermal $\left(\Phi_{\text {epi }}\right)$ and the thermal $\left(\Phi_{\text {them }}\right)$ neutron fluxes in the conditions with and without the cadmium sheet. The value of the gamma-ray dose $\left(D_{\gamma}\right)$ divided by the epithermal neutron flux is also indicated. The gamma-ray dose is almost equal to that of fast neutrons. The main component of the secondary gamma rays is attributable to the neutron capture reaction of cadmium that is employed for thermal neutron shielding. In the absence of cadmium, the gamma-ray dose was confirmed to be almost one order of magnitude lower, as indicated in the table. However, in this condition, the thermal neutron flux increased significantly and it became almost the same order of magnitude as the epithermal flux. We examined the use of boron instead of cadmium for thermal neutron shielding; however, the epithermal neutron flux also decreased significantly and the flux level was unsuitable for the treatment. The dose contribution of fast neutrons and secondary gamma rays should be investigated in greater detail in the future.

Table. 2 Epithermal and thermal neutron fluxes and the value of gamma-ray dose divided by epithermal neutron flux.

\begin{tabular}{|c|c|c|}
\hline $\mathrm{D}_{2} \mathrm{O}$ moderator & With Cd & Without Cd \\
\hline $\begin{array}{c}\Phi_{\text {epi }} \\
{\left[\times 10^{9} / \mathrm{cm}^{2} / \text { source }\right]}\end{array}$ & 2.1 & 2.2 \\
\hline$\Phi_{\text {them }}$ & 0.02 & 2.0 \\
{$\left[\times 10^{9} / \mathrm{cm}^{2} /\right.$ source $]$} & & \\
\hline $\mathrm{D}_{\gamma} / \Phi_{\text {epi }}$ & 2.2 & 0.18 \\
{$\left[\times 10^{-12} \mathrm{~Gy} \mathrm{~cm}^{2}\right]$} & & \\
\hline
\end{tabular}

\section{Conclusion}

For the development of an accelerator neutron source using the $\mathrm{p}-\mathrm{Li}$ reaction, a compact beam shape assembly was designed and neutron and gamma-ray emission characteristics were examined. To obtain a sufficient epithermal flux, the distance between the target and the treatment position should be set less than approximately $25 \mathrm{~cm}$ because otherwise, the neutron source intensity is limited. When the dimensions of the moderator materials are restricted, the use of heavy water as the neutron moderator was found to be the most suitable among the materials we examined. However, in our calculation, fast neutron and secondary gamma-ray doses were found to be still higher, indicating the difficulty in shielding over a small distance.

The initial energy of neutrons generated by the near-threshold $\mathrm{p}$-Li reaction is lower than that of neutrons generated by other methods such as the spallation reaction. This feature is advantageous for reducing the amount of moderator materials required and it enables the building of a compact neutron source. The effective shielding of fast neutrons and gamma rays in a tight space is currently being studied to develop an accelerator neutron source for BNCT.

\section{References}

1) Y. Nakagawa, T. Kobayashi, H. Fukuda., "Advances in Neutron Capture Therapy 2006," Proceedings of ICNCT-12, Takamatsu Kagawa, Japan, Oct. 9-13, 2006 (2006).

2) S. GREEN, "Developments in accelerator based boron neutron capture therapy”, Radiat. Phys. Chem. Vol. 51, No. 4-6 561 (1998).

3) J. F. Briesmeister, Ed., "MCNP-A General Monte Carlo N-Particle Transport Code Version 4C" LA-13709-M, Los Alamos National Laboratory, (2000).

4) C. N. Clubertson, S. Green, et. al, "In-phantom characterization studies at the Birmingham Accelerator-Generated epithermal Neutron Source (BAGINS) BNCT facility", Appl. Rad. And Iso. Vol. 61, Issue 5733 (2004). 
5) T. Nakagawa, K. Shibata, et. al, "Evaluated Nuclear Data Library Version 3 Revision-2: JENDL-3.2", J. Nucl. Sci. Technol., 32, 1259 (1995).

6) M. Drosg, "DROSG-2000:Neutron Source Reactions", IAEA-NDS-87, Rev. 8 (2003).

7) C. L. Lee, X. -L. Zhou, "Thick target neutron yields for the ${ }^{7} \mathrm{Li}(\mathrm{p}, \mathrm{n})^{7} \mathrm{Be}$ reaction near threshold", Nucl. Instr. and Meth. In Phys. Res. B 1521 (1999).

8) H. Miyamaru, I. Murata, Y. Ootera and A. Kitamura, "Measurement of gamma-ray emission from $\mathrm{p}-\mathrm{Li}, \mathrm{p}-\mathrm{Be}$ and d-Be reactions for accelerator-based BNCT", Proceedings of ICNCT-12, Takamatsu Kagawa, Japan, Oct. 9-13,2006 374 (2006). 